\title{
Waiting for the maths
}

Economics is widely regarded as the queen of the social sciences. A few years ago, in his best selling book The End of History, the American political scientist Francis Fukuyama loosely estimated that today's dominant 'neo-classical' theory of economics is, perhaps, $80 \%$ correct. Physicists haven't taken such a sanguine view.

In the wake of the recent financial crisis, a number of physicists - especially those with finance experience - have attacked the mathematics of neo-classical theory for dubious assumptions about human rationality, and for reliance on simple notions of equilibrium in modelling economic reality. Statistical physicist Jean-Philippe Bouchaud, for example, who is also a founding member of a successful hedge fund, argued in Nature that economics needs a scientific revolution. Economists too, including recent Nobel Prize winner Paul Krugman, have suggested that the crisis offered a final, definitive disproof of prevailing neo-classical models of markets as stable, self-regulating systems.

The question is what might replace such theories. Some of the most promising ideas so far seem to come from joining traditional perspectives from economics - in particular, on the market consequences of individual profit-seeking behaviour, or on the role of innovations in spurring economic growth with mathematical models emerging from the study of non-equilibrium systems, especially those involving some kind of evolutionary dynamics. Physicists can't go into economics and arrogantly 'show them how to do it', but they may well contribute by improving the richness of the mathematics that economists use.

The neo-classical view of financial markets is expressed in the so-called efficient-markets hypothesis, which asserts that stocks, for example, should never be either under- or overvalued because markets act very quickly to make the price reflect all information about the underlying company. Through the market mechanism, so says the idea, human greed and rationality can be relied on to keep values at the correct level.

A more realistic alternative to this view is now emerging, in part through the work of financial theorist Andrew Lo. He argues for an 'adaptive-markets hypothesis' that would acknowledge, first, that people often make all kinds of errors of judgement, and second, that markets involve social dynamics and so often follow collective swings that take them, for a time at least, quite far from any equilibrium. Markets, in this view, are far from perfect;

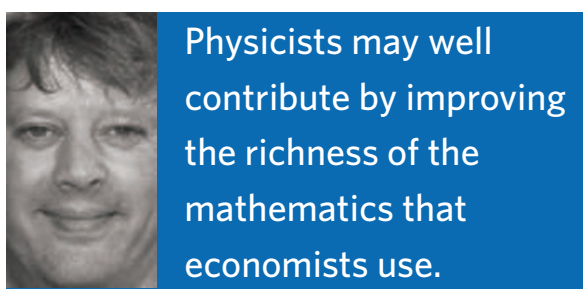

they evolve, learn and adapt, and over time fluctuate (sometimes quite violently) about some more or less sensible position.

Lo argues for this position mostly in words, but some mathematical models express the idea in more specific form. Most notable, perhaps, are those taking a computational approach and which model markets as dynamic ecologies of interacting agents and their strategies. This general class of models - stimulated by initial pioneering collaboration between physicists and economists at the Santa Fe Institute 15 years ago - now reproduce many statistical features of real markets, such as, for example, the clustering of market volatility (reflected in long periods of calm being broken by episodes of violent fluctuation).

But economics goes far beyond financial markets, even if this is where most physics-inspired work has so far tended to focus, perhaps because of the real danger that scientists straying too far past interdisciplinary boundaries can easily end up reinventing the wheel or worse. But this is also changing as a new generation of scientists emerge with training in both physics and economics, and can bring physics ideas to bear on promising economic insights that have just been waiting for the right mathematics to come along.

Recent work by physicist and economist Stefan Thurner and colleagues is a good example (http://arxiv.org/ abs/0909.3482; 2009). They've tried to develop the inspiring ideas of Austrian economist Joseph Schumpeter, who argued that economic activity emerges from a process driven by the appearance and disappearance of goods and services linked to human creativity and technological innovation. The result, he suggested, should be an erratic process of 'creative destruction' as new innovations displace established goods.

Giving Schumpeter's ideas a modern mathematical form is what Thurner et al. have started to do. In their model, existing goods are combined to create new ones, which appear in an economic world already populated by other goods, and threaten the standing of at least some of them. Just as invasive species can trigger cascades of extinctions and rearrangements within an ecosystem, innovative products set off what Schumpeter called "gales of destruction" of varying significance.

This model is necessarily highly schematic, but works with plausible assumptions for the space of possible products, and the dynamics by which they compete. In simulations of the process, this Schumpeterian economy naturally experiences periods of calm and economic stability, but also sporadic tumultuous episodes in which lots of products disappear and the markets shift into wholly new structures.

This kind of model - of the general selforganized-criticality class of dynamics has a feel that is much more naturally in tune with actual market dynamics than anything from general equilibrium theory. An appealing feature is that these waves of creative destruction, even though they arise unpredictably and erratically, stem from relatively ordinary innovations, rather than any external shocks to the system. More significantly, typical time series from the model showing the number of different products, for example, or the rate of business failures or fluctuations in the economy's gross domestic product, all show fat-tailed distributions much like those in real economies.

This is precisely the kind of interplay between specific ideas developed after long observation by 'domain experts' in economics, and new dynamical models coming from physics, that really seems likely to improve our understanding of economic systems. The issue now is perhaps not whether models of this kind will reach the economic mainstream, but when.

Most economists, it seems, still prefer standard equilibrium models, which contain no evolutionary or adaptive dynamics at all, because they can be solved analytically. This is a high price to pay, but at least some in the orthodox old guard seem intent on keeping it that way. In an essay in The Economist, Robert Lucas, one of the key figures behind the present neo-classical theory of macroeconomic systems, even argued that the tumultuous events of the recent crisis can be taken as further evidence supporting the efficient-markets hypothesis of neo-classical theory, despite the fact that it disputes the possible existence of financial bubbles. 